Bangladesh J. Zool. 49(1): 19-34, 2021

ISSN: 0304-9027

eISSN: 2408-8455

\title{
ICTHYOFAUNA OF MADHUMATI RIVER AT MOHAMMADPUR, MAGURA
}

\author{
Md. Anichur Rahman, AHM Shafiullah Habib* \\ Shova Saha and Mallika Saha \\ Department of Zoology, Faculty of Life and Earth Sciences \\ Jagannath University, Dhaka-1100, Bangladesh
}

\begin{abstract}
The investigation was conducted on the present situation of fish biodiversity and abundance of fish fauna at two different points of Madhumati River at Mohammadpur upazilla under Magura district from October 2018 to September 2019. A total of 83 species of fresh water fish species were recorded belonging to 58 genera of 28 families under 11 orders. Cypriniformes was recorded as the dominant order comprising 26 species constituting $31 \%$ of the total. According to IUCN 59\% of fish species were Least Concern, 12\% were Vulnerable, $11 \%$ were Endangered, 9\% were Near Threatened, 1\% of each were Data Deficient and Critically Endangered locally, and 7\% Exotic fish species were also enlisted. Annual value of Shannon diversity index $(\mathrm{H})$ was 1.974 and Sipmson diversity index $(\lambda)$ was 0.272 which indicate highest diversity of fish was in March. Annual Margalef's richness index (D) was 6.654 and Pielou's evenness index (E) was 0.447 denote highest richness was in November and evenness was in March. The river was found to have moderate type of diversity in terms fish species composition.
\end{abstract}

Key words: Madhumati River, fish fauna, diversity status, diversity indices, diversity richness

\section{INTRODUCTION}

Bangladesh is favoured with various inland water bodies endowed with rich diversity in fish species (Islam et al., 2016). It has the third biggest aquatic fish biodiversity in Asia, after China and India, with about 800 species in fresh, brackish and marine waters (Hussain and Mazid, 2001). It enriched with fish fauna support at least 265 freshwater fin fish species under 154 genera and 55 families (Rahman, 2005). More detailed systematic account of the Inland Fisheries of the Indian Region and Adjacent countries including Bangladesh has been made by Talwar and Jhingran (1991). Bangladesh is one of the world's prominent fish producing country with a total production of 4.276 million MT in FY 2017-18 (DoF, 2018). Through this remarkable achievement Bangladesh for the first time in the history has become self-sufficient in fish production

* Author for corresponding: <ahmshabib@gmail.com>

(c)2021 Zoological Society of Bangladesh DOI: https://doi.org/10.3329/bjz.v49i1.53679 
providing $62.58 \mathrm{~g}$ of fish per person in everyday dietary consumption. Last 10 years average growth performance of this sector is $5.26 \%$, which seems quite consistent and encouraging (DoF, 2018).

Madhumati is a very important river in Bangladesh having very wide and extensive. It is also one of the longest rivers in Bangladesh and a distributary of the Ganges flows through Kushtia, Magura, Jessore, Faridpur, Pirojpur and Khulna districts in the country. The river has been named as the Gorai in the upper part and Madhumati in the lower. The Gorai River started its course at Talbaria, north of Kushtia town and $19 \mathrm{~km}$ downstream from Hardinge Bridge. South of Kushtia its first branch, the Kaliganga joined with the Kumar River near Shailkupa. The main river is divided and rejoined several times as it flows southeast to Mohammadpur upazila under Magura district. From this point it changes its name to Madhumati (Banglapedia, 2014).

Works on status of freshwater fish and diversity of fish in Bangladesh have been done by Shafi and Quddus (1982), Hossain et al. (2013), Galib et al. (2013), Joadder et al. (2015), Islam et al. (2016) and Easmin et al. (2018), whereby checklist of fish, showing conservation status of fish and diversity of fish was made. IUCN Red list of Bangladesh (2015) reported a total of 253 fish species were assessed of which 64 species have been found threatened consisting $25.3 \%$ of the total species. Among them 9 species were evaluated as Critically Endangered (CR), 30 species as Endangered (EN), 25 species as Vulnerable (VU), 27 species as Near Threatened (NT), 122 species as Least Concern (LC) and the rest 40 species were considered Data Deficient (DD). No fish was identified as extinct or regionally extinct.

The deterioration of natural water bodies resulting from human interference due to construction of roads, deforestation, embankments, invasion to agricultural lands, indiscriminate use of pesticides and natural causes had negative impact of fish diversity in the river. It was selected for its contribution in fisheries sectors. Fish status in the river may impact on the society of the river area. The rich diversity may elucidate further fish resources in the area which may ensure the protein supply of the local people. Instead, the indiscriminate harmful techniques of fishing, use of different fishing gears threatens the biodiversity of the seasonal floodplains. In case of less diversity awareness program may be initiated alongside the river for the development of the fish fauna of the river. Hence, there is necessity to update the species checklist of fish available in the Madhumati River and present status of the fish and 
their diversity, richness and evenness in the river. This exploration was aimed to build up authentic information about the species available at the Madhumati River in south-west part of country.

\section{MATERIAL AND METHODS}

The Madhumati River was selected for this study to assess the fish fauna and their current status. The fish samples were collected monthly from the river during October 2018 to September 2019. Two points were selected in Magura District at Mohammadpur Upazila, which were Elangkhali Ghat $\left(23^{\circ} 24^{\prime} 29.7^{\prime \prime} \mathrm{N}\right.$

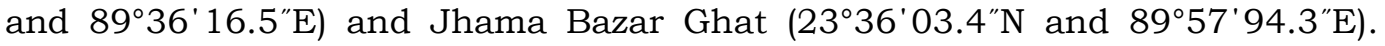
Monthly fish samples were collected from the selected spots directly from the fishermen, retailers, 'Nikari', 'Bapari' and 'Aratdar' from the two preselected sites. Some fishes were directly identified in the sampling spots and rest of the fish specimens were carried in ice box or safeguarded in 4 to $6 \%$ buffered formalin solution to the Fisheries Laboratory, Department of Zoology, Jagannath University for identification and further taxonomic study. Large fishes were easily counted and recorded but small fishes were counted using the following formula (Iqbal et al. 2015):

$N=N s \times \frac{W t}{W s}$

Where, $N$ is the total number of a fish species, $N s$ is number of individual small fish in each subsample, Wt is the total weight of small fish found in the sample and $W s$ is weight of subsample. Fish species were identified based on their morphometric and meristic characters following Fish Base (2020), Rahman (2005), Talwar and Jhingran (1991) and Bhuiyan (1964). Identified fishes were systematically arranged according to Nelson (2006). Fish status were categorized according to IUCN Redlist (2015, Volume 5: Freshwater Fish). Diversity, richness and evenness were calculated using the following formulae:

Shannon-Weaver diversity index, $H=-\Sigma P i \ln P i$ (Shannon-Weaver, 1949)

Simpson index, $\lambda=\Sigma P i^{2}$ (Simpson, 1949)

Margalef's richness index, $D=\frac{(S-1)}{\ln (N)}$ (Margalef, 1968)

Pielou's evenness index, $E=\frac{H}{\ln (S)}$ (Pielou, 1966)

Where, $H$ and $A$ is the diversity index, $P i$ is the relative abundance $(n / N),, \mathrm{n}$ is the number of individual for each species, $N$ is the total number of individual, $D$ 
is the richness index, $E$ is the similarity or evenness index, $S$ is the total number of species and $\ln$ is the natural logarithm.

Statistical analysis was done with help of Microsoft Office Excel 2016.

\section{RESULTS AND DISCUSSION}

A total 83 species under 58 genera were identified belonging to 28 families under 11 orders (Table 1, Fig 7 - 89). The most dominant order was Cypriniformes comprising $31 \%$ of all the number of species recorded and the number of fish species was 26. Other dominating orders were Perciformes, Siluriformes, Clupeiformes and Channiformes which comprised 20,19, 6 and 4 species of fish respectively. The lowest numbers of fish species representing orders were Anguiliformes, Pleuronectiformes, Synbranchiformes and Tetraodontiformes that consisted only 1 species of fish each (Fig. 1). Galib et al. (2013) reported on the fish fauna of Choto Jamuna River and a total of 63 species of fishes had been recorded under 41 genera, 23 families and 9 orders. The most dominant order was Cypriniformes comprising 34.92\%, next to Cypriniformes, other dominant orders were Siluriformes, Perciformes and Synbranchiformes constituting $28.57 \%, 19.05 \%$ and $6.35 \%$ of species recorded respectively. Islam et al. (2016) found Perciformes as most dominant order constituting $42.62 \%$ of the total fish population followed by Siluriformes (11.48\%), Clupeiformes (9.84\%), Pleuronectiformes (3.29\%), Synbranchiformes (3.29\%), Osteoglossiformes (1.64\%), Beloniformes (1.64\%), Mugiliformes (4.92\%), Cypriniformes (16.39\%) and Tetraodontiformes (4.92\%) in the Sibsa River in South-Western Bangladesh.

In family composition, the most dominant family was Cyprinidae comprising 22 species under 12 genera. The minimal number (1 species) belonged to 11 families of fish viz. Ophichthidae, Pangasiidae, Synbranchidae, Heteropneustidae, Nandidae, Mugilidae, Anabantidae, Cynoglossidae, Belonidae, Hemiramphidae, Tetraodontidae (Fig. 2). Azadi and Alam (2013) reported Cyprinidae as the most dominant family comprising 20 species and other dominant families were Gobiidae, Schilbeidae and Bagridae constituting 11, 5 and 4 species respectively in the Halda River.

According to IUCN (2015), 59\% of fish species were Least Concern, 12\% were Vulnerable, 11\% were Endangered, 9\% were Near Threatened and 1\% was Data Deficiency and Critically Endangered and 7\% Exotic species were also recorded (Fig. 3). Easmin et al. (2018) reported 77 species of fish in the Jamuna River where 59\% was Least Concern (LC), 23\% were Threatened including 1\% Critically Endangered (CR), 12\% Endangered (EN) and 10\% Vulnerable (VU) species, and 5\% Exotic fish was also recorded during the study period. 
Table 1. Fish species of Madhumati River of Magura

\begin{tabular}{|c|c|c|c|c|c|c|}
\hline Order & Family & Scientific Name & $\begin{array}{r}\text { Local } \\
\text { Name }\end{array}$ & $\begin{array}{c}\text { English } \\
\text { Name }\end{array}$ & $\begin{array}{l}\text { Local } \\
\text { Status } \\
\end{array}$ & $\begin{array}{l}\text { Global } \\
\text { Status } \\
\end{array}$ \\
\hline \multirow[t]{2}{*}{ Osteoglossiformes } & Notopteridae & 1. Chitala chitala & Chital & $\begin{array}{l}\text { Clown knife } \\
\text { fish }\end{array}$ & EN & NT \\
\hline & & 2. Notopterus notopterus & Foli & $\begin{array}{l}\text { Bronze feather } \\
\text { back }\end{array}$ & VU & LC \\
\hline Anguiliformes & Ophichthidae & 3. Pisodonophis boro & Kharu, Hijra & $\begin{array}{l}\text { Rice paddy } \\
\text { eel }\end{array}$ & $\mathrm{LC}$ & LC \\
\hline Synbranchiformes & Synbranchidae & 4. Monopterus cuchia & $\begin{array}{l}\text { Kuchia, } \\
\text { Kuicha }\end{array}$ & Cuchia & VU & VU \\
\hline \multirow[t]{6}{*}{ Clupeiformes } & Clupeidae & 5. Corica soborna & Kachki & $\begin{array}{l}\text { Ganges river } \\
\text { spral }\end{array}$ & LC & LC \\
\hline & & 6. Pellona ditchela & Choukka & Indian pellona & LC & NE \\
\hline & & 7. Tenualosa ilisha & llish & Hilsha shad & LC & LC \\
\hline & Engraulidae & 8. Gudusia chapra & Chapila & $\begin{array}{l}\text { Indian river } \\
\text { shad }\end{array}$ & VU & $\mathrm{LC}$ \\
\hline & & 9. Setipinna phasa & Phasa & $\begin{array}{l}\text { Gangatic } \\
\text { hairfin } \\
\text { anchovy }\end{array}$ & LC & LC \\
\hline & & 10. Setipinna taty & Teli phasa & $\begin{array}{l}\text { Scaly hairfin } \\
\text { anchovy }\end{array}$ & $\mathrm{LC}$ & NE \\
\hline \multirow[t]{4}{*}{ Channiformes } & Channidae & 11. Channa marulius & Gajar & $\begin{array}{l}\text { Great } \\
\text { snakehead }\end{array}$ & EN & LC \\
\hline & & 12. Channa orientalis & Cheng & $\begin{array}{l}\text { Walking } \\
\text { snakehead }\end{array}$ & LC & LC \\
\hline & & 13. Channa puncta & Taki & $\begin{array}{l}\text { Spotted } \\
\text { snakehead }\end{array}$ & $\mathrm{LC}$ & $\mathrm{LC}$ \\
\hline & & 14. Channa striata & Shol & $\begin{array}{l}\text { Snakehead } \\
\text { murrel }\end{array}$ & LC & LC \\
\hline \multirow[t]{18}{*}{ Cypriniformes } & Cyprinidae & 15. Amblypharyngodon mola & Mola & Mola carplet & LC & LC \\
\hline & & 16. Aspidoparia morar & $\begin{array}{l}\text { Morar, } \\
\text { Morari }\end{array}$ & Aspidopara & VU & NE \\
\hline & & 17. Barbonymus gonionotus & $\begin{array}{l}\text { Thai } \\
\text { sarpunti }\end{array}$ & Java barb & Exotic & Exotic \\
\hline & & 18. Catla catla & Katla & Catla & LC & NE \\
\hline & & 19. Cirrhinus cirrhosus & Mrigal & Mrigal crap & NT & VU \\
\hline & & 20. Cirrhinus reba & Tatkini & Reba & NT & LC \\
\hline & & $\begin{array}{l}\text { 21. Cyprinus carpio var. } \\
\text { carpio }\end{array}$ & $\begin{array}{l}\text { Common } \\
\text { carp }\end{array}$ & Common carp & Exotic & Exotic \\
\hline & & $\begin{array}{l}\text { 22. Cyprinus carpio var. } \\
\text { nudus }\end{array}$ & Carpu & Common carp & Exotic & Exotic \\
\hline & & 23. Esomus danricus & Darkina & Flying barb & LC & LC \\
\hline & & $\begin{array}{l}\text { 24. Hypophthalmichthys } \\
\text { molitrix }\end{array}$ & Silver carp & Silver carp & Exotic & Exotic \\
\hline & & $\begin{array}{l}\text { 25. Hypophthalmichthys } \\
\text { nobilis }\end{array}$ & $\begin{array}{l}\text { Big-head } \\
\text { carp }\end{array}$ & Big-head carp & Exotic & Exotic \\
\hline & & 26. Labeo bata & Bata & Bata labeo & LC & $\mathrm{LC}$ \\
\hline & & 27. Labeo calbasu & Kalibaus & $\begin{array}{l}\text { Orange fin } \\
\text { labeo }\end{array}$ & LC & LC \\
\hline & & 28. Labeo rohita & Rui & Rohu & LC & LC \\
\hline & & 29. Osteobrama cotio & $\begin{array}{l}\text { Lohasur, } \\
\text { Keti }\end{array}$ & Cotia & NT & $\mathrm{LC}$ \\
\hline & & 30. Pethia conchonius & $\begin{array}{l}\text { Kanchan } \\
\text { punti }\end{array}$ & Rosy barb & LC & LC \\
\hline & & 31. Pethia guganio & Mola punti & Glass barb & LC & $\mathrm{LC}$ \\
\hline & & 32. Pethia ticto & Tit punti & Ticto barb & VU & $\mathrm{LC}$ \\
\hline
\end{tabular}




\begin{tabular}{|c|c|c|c|c|c|c|}
\hline Order & Family & Scientific Name & $\begin{array}{l}\text { Local } \\
\text { Name }\end{array}$ & $\begin{array}{c}\text { English } \\
\text { Name }\end{array}$ & $\begin{array}{l}\text { Local } \\
\text { Status }\end{array}$ & $\begin{array}{l}\text { Globa } \\
\text { Status }\end{array}$ \\
\hline & & $\begin{array}{l}\text { 33. Puntius chola } \\
\text { 34. Puntius sophore }\end{array}$ & $\begin{array}{l}\text { Chala punti } \\
\text { Jat punti }\end{array}$ & $\begin{array}{l}\text { Swamp barb } \\
\text { Pool barb }\end{array}$ & $\begin{array}{l}\mathrm{LC} \\
\mathrm{LC}\end{array}$ & $\begin{array}{l}\mathrm{LC} \\
\mathrm{LC}\end{array}$ \\
\hline & & 35. Salmostoma bacaila & $\begin{array}{l}\text { Narkali } \\
\text { chela }\end{array}$ & $\begin{array}{l}\text { Large } \\
\text { razorbelly } \\
\text { minnow }\end{array}$ & LC & LC \\
\hline & & 36. Salmostoma phulo & Fulchela & $\begin{array}{l}\text { Finescale } \\
\text { razorbelly } \\
\text { minnow }\end{array}$ & NT & LC \\
\hline & Cobitidae & 37. Botia dario & Rani & Bengal loach & EN & LC \\
\hline & & 38 . Botia lohachata & Putul & $\begin{array}{l}\text { Reticulate } \\
\text { loach }\end{array}$ & EN & NE \\
\hline & & $\begin{array}{l}\text { 39. Lepidocephalichthys } \\
\text { annandalei }\end{array}$ & Gutum & $\begin{array}{l}\text { Annandale } \\
\text { loach }\end{array}$ & VU & LC \\
\hline & & $\begin{array}{l}\text { 40. Lepidocephalichthys } \\
\text { guntea }\end{array}$ & Gutum & Guntea loach & LC & LC \\
\hline \multirow[t]{19}{*}{ Siluriformes } & Bagridae & 41. Mystus bleekeri & $\begin{array}{l}\text { Gulsha } \\
\text { tengra }\end{array}$ & Day's mystus & LC & LC \\
\hline & & 42. Mystus cavasius & $\begin{array}{l}\text { Kabashi } \\
\text { tengra }\end{array}$ & $\begin{array}{l}\text { Gangetic } \\
\text { mystus }\end{array}$ & NT & LC \\
\hline & & 43. Mystus tengara & $\begin{array}{l}\text { Bajari } \\
\text { tengra }\end{array}$ & $\begin{array}{l}\text { Stripped dwarf } \\
\text { catfish }\end{array}$ & LC & LC \\
\hline & & 44. Mystus vittatus & Tengra & $\begin{array}{l}\text { Asian striped } \\
\text { catfish }\end{array}$ & LC & LC \\
\hline & & 45. Rita rita & Rita & Rita & EN & LC \\
\hline & & 46. Sperata aor & Ayre & $\begin{array}{l}\text { Long- } \\
\text { whiskered } \\
\text { catfish }\end{array}$ & VU & LC \\
\hline & & 47. Sperata seenghala & Guijja, Ayre & $\begin{array}{l}\text { Giant river } \\
\text { catfish }\end{array}$ & VU & LC \\
\hline & Siluridae & 48. Ompok pabda & $\begin{array}{l}\text { Madhu } \\
\text { pabda }\end{array}$ & $\begin{array}{l}\text { Pabdah } \\
\text { catfish }\end{array}$ & EN & NT \\
\hline & & 49. Wallago attu & Boal & $\begin{array}{l}\text { Freshwater } \\
\text { shark }\end{array}$ & VU & NT \\
\hline & Schilbeidae & 50. Ailia coila & $\begin{array}{l}\text { Kajuli, } \\
\text { Baspata }\end{array}$ & Gangetic ailia & LC & NT \\
\hline & & 51. Clupisoma garua & Ghaura & Garua bacha & EN & NE \\
\hline & & 52. Eutropiichthys murius & Muri bacha & Murius vacha & LC & LC \\
\hline & & 53. Eutropiichthys vacha & Bacha & $\begin{array}{l}\text { Batchwa } \\
\text { vacha }\end{array}$ & LC & LC \\
\hline & Pangasiidae & 54. Pangasius pangasius & Pangas & Pungas catfish & EN & LC \\
\hline & Sisoridae & 55. Bagarius bagarius & Baghair & Devil catfish & CR & NT \\
\hline & & 56. Gagata cenia & Cenia & Indian gagata & LC & LC \\
\hline & Clariidae & 57. Clarias batrachus & Magur & $\begin{array}{l}\text { Walking } \\
\text { catfish }\end{array}$ & LC & LC \\
\hline & & 58. Clarias gariepinus & $\begin{array}{l}\text { African } \\
\text { magur }\end{array}$ & $\begin{array}{l}\text { North african } \\
\text { catfish }\end{array}$ & Exotic & Exotic \\
\hline & Heteropneustidae & 59. Heteropneustes fossilis & Shing & $\begin{array}{l}\text { Stinging } \\
\text { catfish }\end{array}$ & LC & LC \\
\hline \multirow[t]{6}{*}{ Perciformes } & Ambassidae & 60. Chanda nama & $\begin{array}{l}\text { Nama } \\
\text { Chanda }\end{array}$ & $\begin{array}{l}\text { Elongated } \\
\text { glass-perchlet }\end{array}$ & LC & LC \\
\hline & & 61. Parambassis lala & Lal chanda & $\begin{array}{l}\text { Highfin glassy } \\
\text { perchlet }\end{array}$ & LC & $\mathrm{NE}$ \\
\hline & & 62. Parambassis ranga & $\begin{array}{l}\text { Ranga } \\
\text { chanda }\end{array}$ & $\begin{array}{l}\text { Indian glassy } \\
\text { fish }\end{array}$ & LC & LC \\
\hline & Sciaenidae & 63. Johnius coitor & Koitor poa & Coitor croaker & LC & LC \\
\hline & & 64. Otolithoides pama & Poa & Pama croaker & LC & NE \\
\hline & Nandidae & 65. Nandus nandus & Bheda, & Mottled & NT & LC \\
\hline
\end{tabular}




\begin{tabular}{|c|c|c|c|c|c|c|}
\hline Order & Family & Scientific Name & $\begin{array}{l}\text { Local } \\
\text { Name }\end{array}$ & $\begin{array}{c}\text { English } \\
\text { Name }\end{array}$ & $\begin{array}{l}\text { Local } \\
\text { Status }\end{array}$ & $\begin{array}{l}\text { Global } \\
\text { Status }\end{array}$ \\
\hline & & & Meni & nandus & & \\
\hline & Mugilidae & 66. Rhinomugil corsula & Khorsula & Corsula mullet & LC & LC \\
\hline & Gobiidae & 67. Eugnathogobius oligactis & Bele & Goby & VU & LC \\
\hline & & 68. Glossogobius giuris & Bele & Tank goby & LC & LC \\
\hline & & 69. Oxyurichthyes microlepis & Nuna baila & $\begin{array}{l}\text { Small scaled } \\
\text { goby }\end{array}$ & LC & NE \\
\hline & & $\begin{array}{l}\text { 70. Pseudapocryptes } \\
\text { elongatus }\end{array}$ & Chewa & $\begin{array}{l}\text { Lanceolate } \\
\text { goby }\end{array}$ & LC & LC \\
\hline & Gobioididae & 71. Eleotris lutea & Kuli, Goby & Lutea sleeper & $\mathrm{DD}$ & NE \\
\hline & & $\begin{array}{l}\text { 72. Odontamblyopus } \\
\text { rubicundus }\end{array}$ & Lal chewa & $\begin{array}{l}\text { Rubicundus } \\
\text { Eelgoby }\end{array}$ & LC & $\mathrm{NE}$ \\
\hline & Anabantidae & 73. Anabas testudineus & Koi & $\begin{array}{l}\text { Climbing } \\
\text { perch }\end{array}$ & LC & $\mathrm{DD}$ \\
\hline & Osphronemidae & 74. Trichogaster chuna & $\begin{array}{l}\text { Chuna } \\
\text { khailsha }\end{array}$ & Dwarf gourami & LC & LC \\
\hline & & 75. Trichogaster fasciatus & Khailsha & $\begin{array}{l}\text { Banded } \\
\text { gourami }\end{array}$ & LC & LC \\
\hline & & 76. Trichogaster lalia & $\begin{array}{l}\text { Lal } \\
\text { Khailsha }\end{array}$ & Dwarf gourami & LC & LC \\
\hline & Mastacembelidae & 77. Macrognathus aculeatus & Tara baim & $\begin{array}{l}\text { Lesser spiny } \\
\text { eel }\end{array}$ & NT & NE \\
\hline & & 78. Macrognathus pancalus & Guchi baim & $\begin{array}{l}\text { Barred spiny } \\
\text { eel }\end{array}$ & LC & LC \\
\hline & & 79. Mastacembelus armatus & Sal baim & Zig-zag eel & EN & NE \\
\hline Pleuronectiformes & Cynoglossidae & 80. Paraplagusia bilineata & Kukur jeeb & $\begin{array}{l}\text { Fingerlip } \\
\text { tonguesole }\end{array}$ & LC & NE \\
\hline \multirow[t]{2}{*}{ Beloniformes } & Belonidae & 81. Xenentodon cancila & Kakila & $\begin{array}{l}\text { Freshwater } \\
\text { garfish }\end{array}$ & LC & NE \\
\hline & Hemirhamphidae & 82. Hyporhamphus limbatus & Ek thuitta & $\begin{array}{l}\text { Congaturi } \\
\text { halfbeak }\end{array}$ & LC & $\mathrm{NE}$ \\
\hline Tetraodontiformes & Tetraodontidae & 83. Leiodon cutcutia & Tepa & $\begin{array}{l}\text { Ocellated } \\
\text { puffer fish }\end{array}$ & LC & LC \\
\hline
\end{tabular}

$\mathrm{CR}=$ Critically Endangered, $\mathrm{EN}=$ Endangered, $\mathrm{VU}=$ Vulnerable, $\mathrm{NT}=$ Near Threatened, $\mathrm{LC}=$ Least Concern, $\mathrm{DD}=$ Data Deficient, $\mathrm{NE}=$ Not Evaluated.

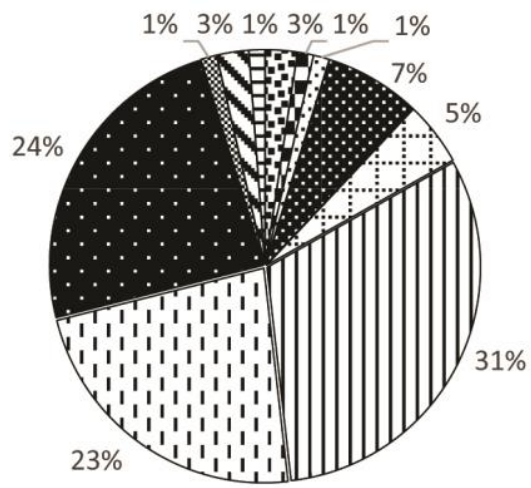

[Osteoglossiformes

anguiliformes

घSynbranchiformes

Clupeiformes

ఐChanniformes

๓Cypriniformes

QSiluriformes

- Perciformes

冈Pleuronectiformes

口Beloniformes

日Tetraodontiformes

Fig. 1. Species composition in different orders of fish. 


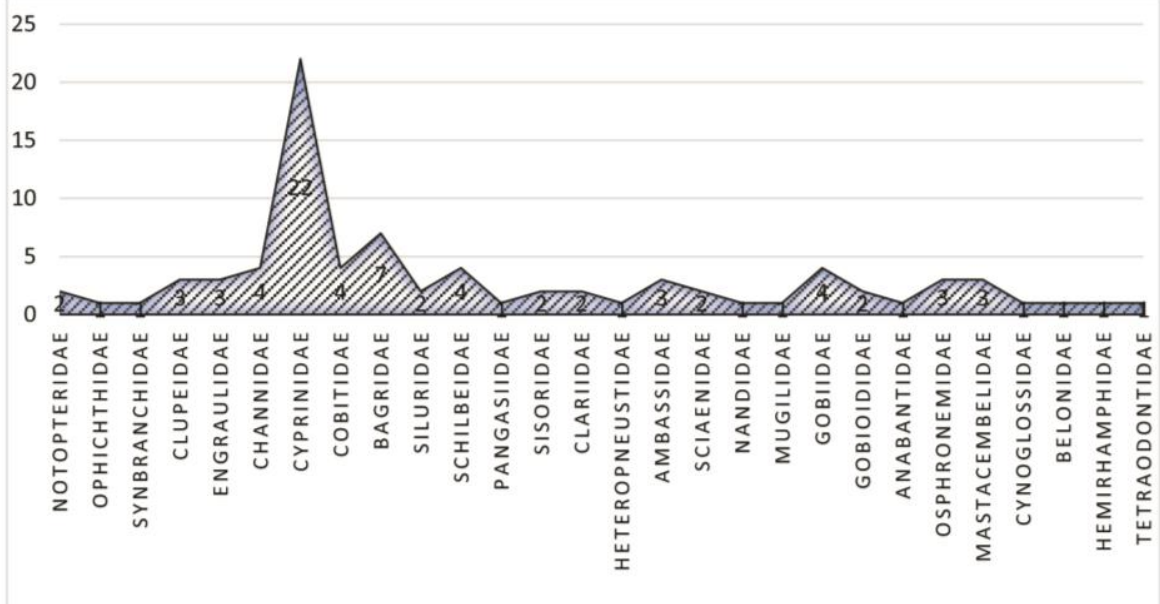

Fig. 2. Species composition of different families of fish in Madhumati River.

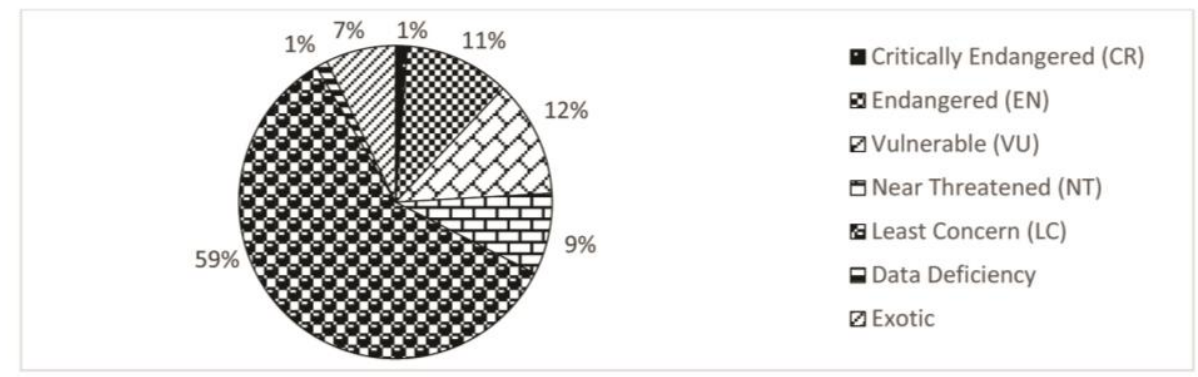

Fig. 3. Local status of fish fauna in the Madhumati River

A total 25 species of fish were found as Threatened locally where the highest number 6 representing the Siluriformes following 5 representing the Cypriniformes (Fig. 4). The lowest number of Threatened species was 1 in each order of Anguiliformes, Synbracchiformes, Beloniformes, Pleuronectiformes and Tetraodontiformes (Fig. 4). In Halda River among 83 species 3 were Critically Endangered and 8 were Vulnerable (Azadi and Alam, 2013).

On global context, 53 (64\%) species of fish were Least Concern, 16 (19\%) were Not Evaluated, 5 (6\%) were Near Threatened, 2 (3\%) were Vulnerable and 1 (1\%) was Data Deficiency. Six (7\%) Exotic species were also recorded in this study (Fig. 5). Joadder et al. (2015) working on Padma River found $72 \%$ of the total fish species to be Least Concern and global conservation status was not assessed by IUCN of $13 \%$ species. 


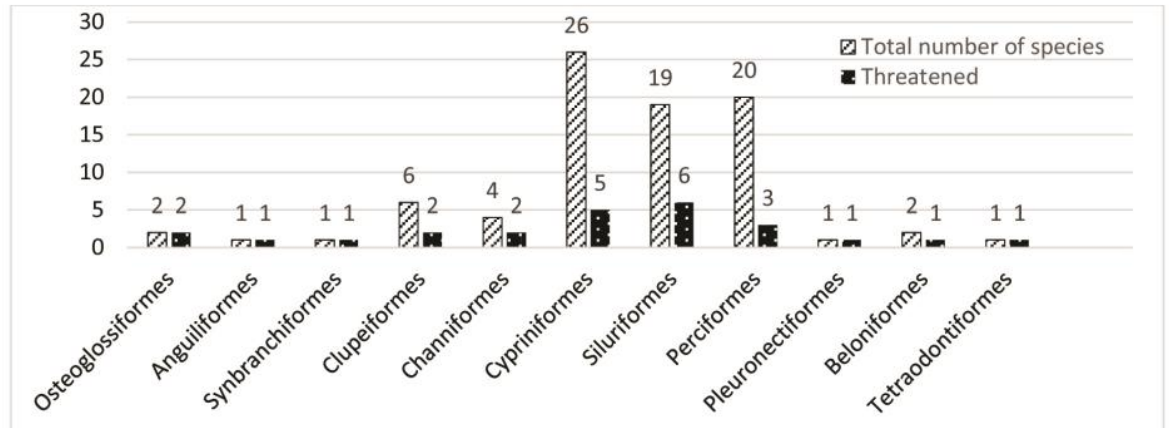

Fig. 4. Order-wise locally threatened fish species of Madhumati River.
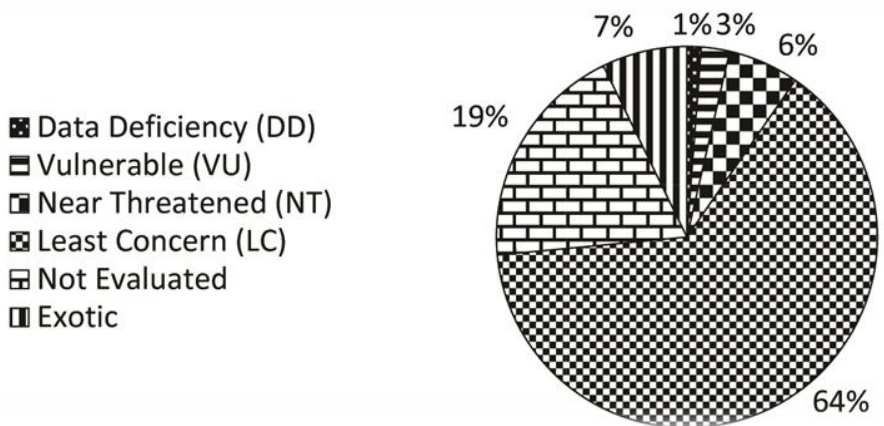

Fig. 5. Global status of fish fauna of Madhumati River.

According to Global status, 4 species of fish were recorded as Threatened among them 2 belonged to the order Perciformes and 1 of each species was found under orders Cypriniformes and Siluriformes (Fig. 6). According to Global status, two species of each were Threatened belonging to Cypriniformes and Synbranchiformes, as reported by Easmin et al. (2018) in the Jamuna River.

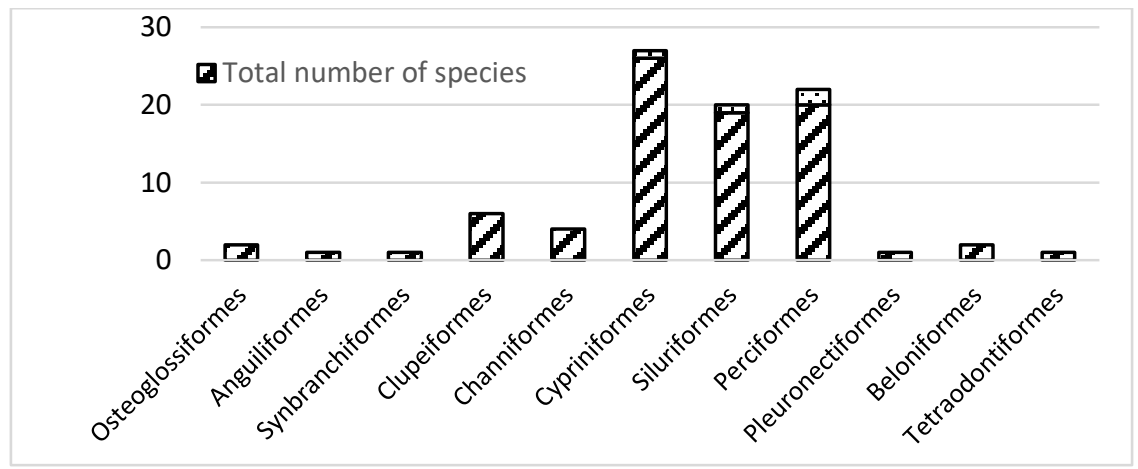

Fig. 6. Globally threatened fish species in different orders of Madhumati River. 
Diversity, Richness and Evenness indices of fishes in Madhumati River: Higher Shannon diversity index was found in March 2.651 and lower 1.369 in January (Table 2). The higher Shannon diversity index value indicates the higher species diversity of the area. Thus in the river highest diversity

Table 2. Different indices of fish fauna of Madhumati River

\begin{tabular}{lcccccc}
\hline \multicolumn{1}{c}{ Months } & $\begin{array}{c}\text { Number } \\
\text { of } \\
\text { species }\end{array}$ & $\begin{array}{c}\text { Number of } \\
\text { Individuals }\end{array}$ & $\begin{array}{c}\text { Shannon } \\
\text { diversity } \\
\text { index (H) }\end{array}$ & $\begin{array}{c}\text { Simpson } \\
\text { diversity } \\
\text { index ( })\end{array}$ & $\begin{array}{c}\text { Margalef's } \\
\text { richness } \\
\text { index (D) }\end{array}$ & $\begin{array}{c}\text { Pielou's } \\
\text { evenness } \\
\text { index (E) }\end{array}$ \\
\hline October'18 & 38 & 2489 & 2.177 & 0.180 & 4.732 & 0.594 \\
November'18 & 48 & 19913 & 2.099 & 0.110 & 4.747 & 0.549 \\
December'18 & 34 & 29526 & 1.480 & 0.336 & 3.206 & 0.419 \\
January'19 & 34 & 16058 & 1.369 & 0.429 & 3.407 & 0.388 \\
February'19 & 36 & 7476 & 1.920 & 0.245 & 3.924 & 0.531 \\
March'19 & 41 & 26184 & 2.651 & 0.099 & 3.932 & 0.629 \\
April'19 & 33 & 18553 & 1.700 & 0.277 & 3.256 & 0.486 \\
May'19 & 32 & 18394 & 1.568 & 0.282 & 3.157 & 0.452 \\
June'19 & 32 & 46463 & 1.393 & 0.313 & 2.884 & 0.402 \\
July'19 & 36 & 11781 & 1.890 & 0.238 & 3.734 & 0.525 \\
August'19 & 36 & 10120 & 1.738 & 0.298 & 3.795 & 0.483 \\
September'19 & 40 & 17841 & 1.631 & 0.351 & 3.984 & 0.441 \\
Annual & 83 & 224798 & 1.974 & 0.272 & 6.654 & 0.447 \\
\hline
\end{tabular}

was seen in March and lowest diversity of fish was in January in the river. Highest Simpson index was 0.429 in January and lowest 0.099 in March (Table 2). Simpson index value ranges between 0 to 1 and low value represents higher diversity. So, according to Simpson's index highest diversity was observed in March and lowest in January.

Highest Margalef's Richness value was 4.747 in November and lowest in 2.884 June (Table 2). Margalef's richness index value depends on species number. Higher the species number, it expresses higher richness in that ecosystem. Highest species number was found in November and lowest species number was found in June. So, November was the highest richness and June was the lowest richness month in term of species richness.

Highest Pielou's Evenness was 0.629 in March and lowest was 0.388 in January. Pielou's evenness index value range from 0 to 1 , if the value is towards 1 , shows species number to be more evenly distributed. Therefore, in March the species were more evenly distributed and less evenly distributed in January.

Annual Shannon diversity index, Simpson diversity index, Richness index, Evenness Index values were $1.974,0.272,6.654$ and 0.447 respectively (Table 2). Annual values of indices shown moderately diversified, Richest and evenly 


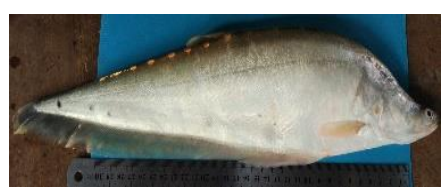

7. Chitala chitala

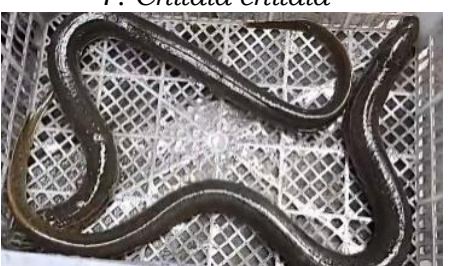

10. Monopterus cuchia

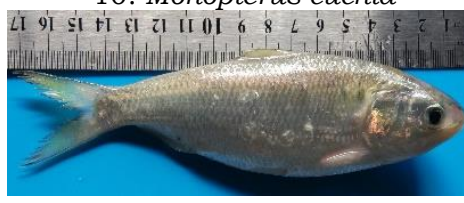

13. Tenualosa ilisha

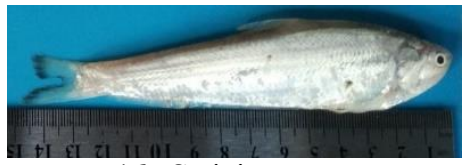

16. Setipinna taty

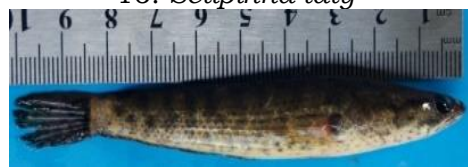

19. Channa punctata

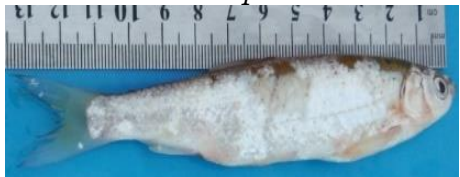

22. Aspidoparia morar

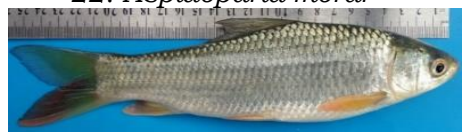

25. Cirrhinus cirrhosus

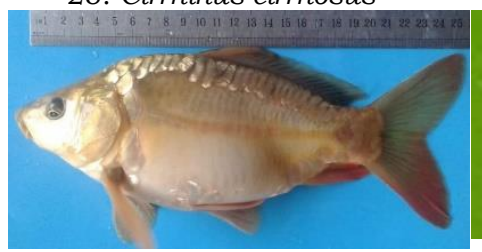

28. Cyprinus carpio var. nudus

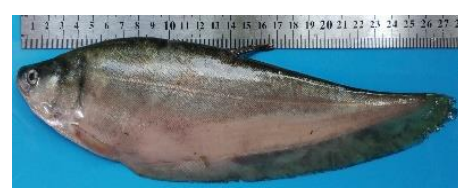

8. Notopterus notopterus

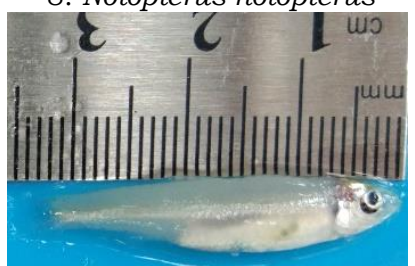

11. Corica soborna

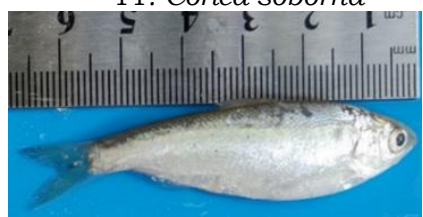

14. Gudusia chapra

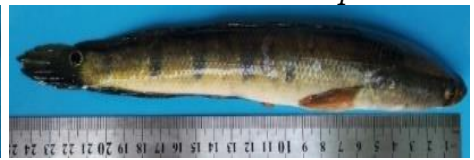

17. Channa marulius

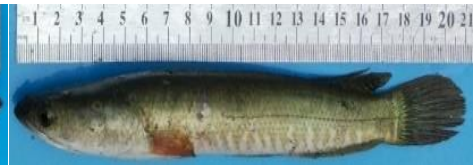

20. Channa striata

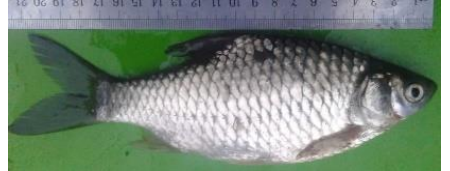

23. Barbonymus gonionotus

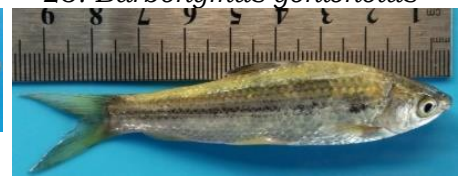

26. Cirrhinus reba

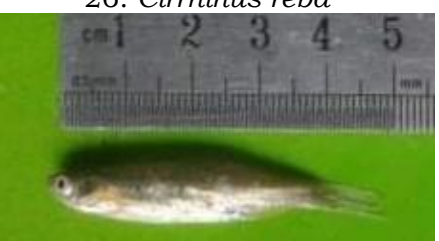

29. Esomus danricus

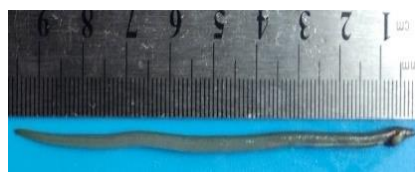

9. Pisodonophis boro

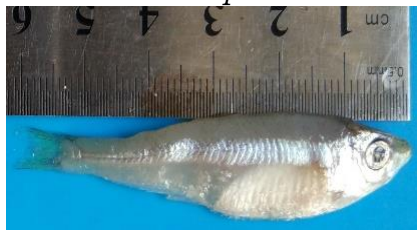

12. Pellona ditchela

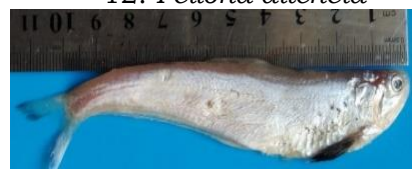

15. Setipinna phasa

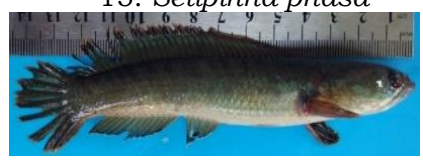

18. Channa orientalis

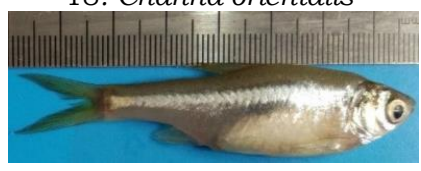

21. Amblypharyngodon mola

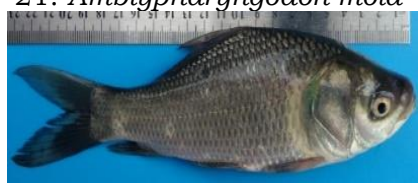

24. Catla catla

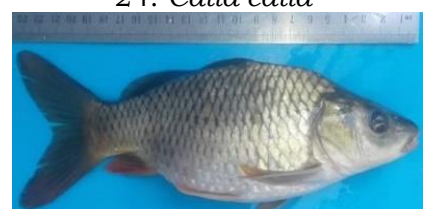

27. Cyprinus carpio var. carpio

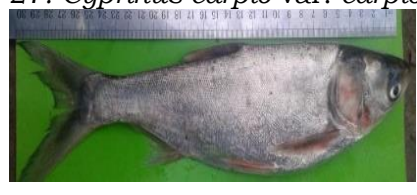

30. Hypophthalmichthys molitrix 

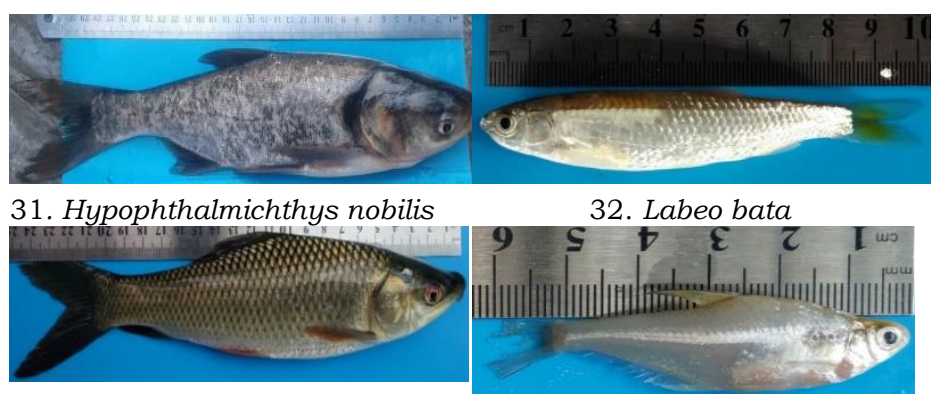

34. Labeo rohita

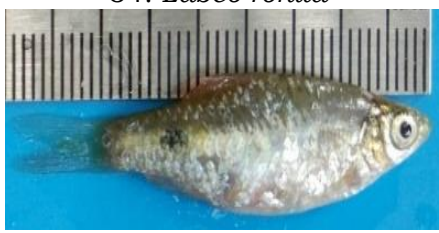

37. Pethia guganio

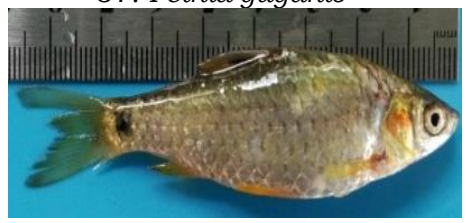

40. Puntius sophore

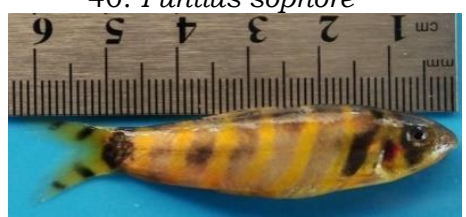

43. Botia dario

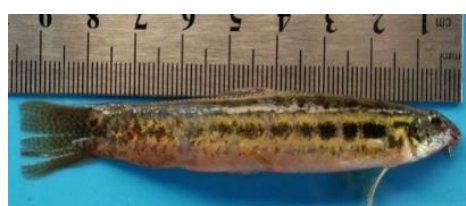

46. Lepidocephalichthys guntea

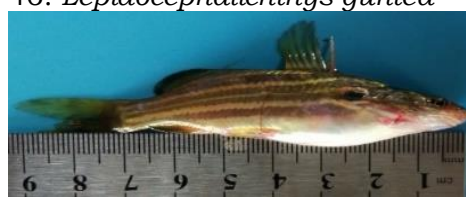

49. Mystus tengara

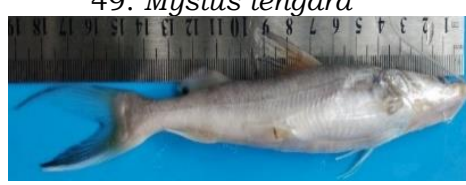

52. Sperata aor
32. Labeo bata

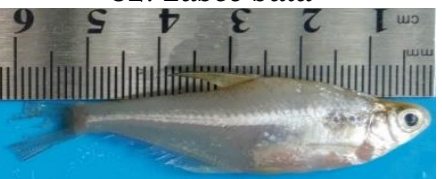

35. Osteobrama cotio

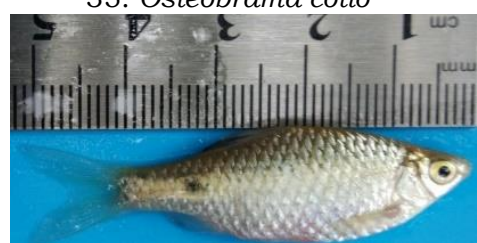

38. Pethia ticto

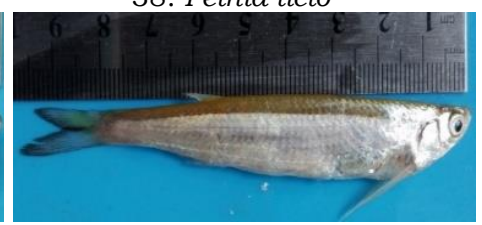

41. Salmostoma bacaila

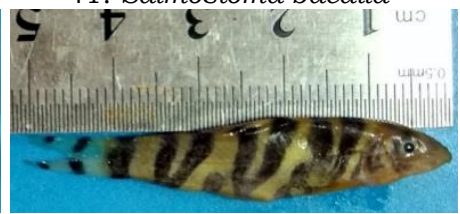

44. Botia lohachata
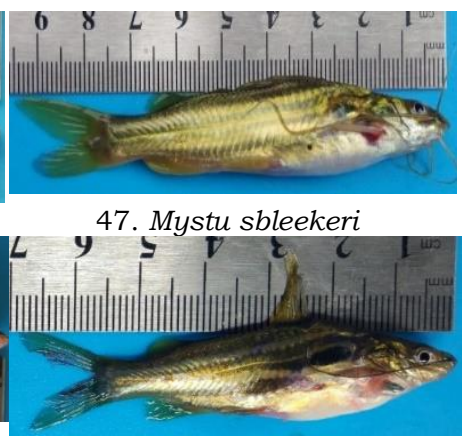

50. Mystus vittatus

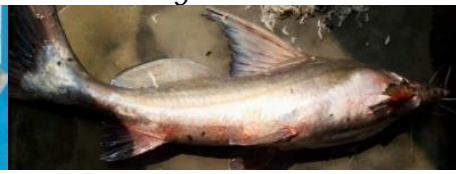

53. Sperata seenghala

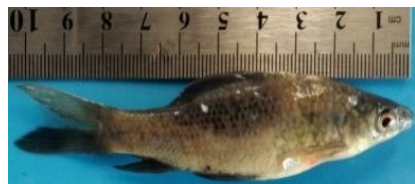

33. Labeo calbasu

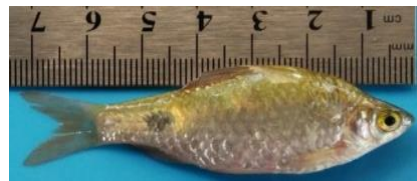

36. Pethia conchonius $8<9 \varsigma \leftarrow \varepsilon \tau$ I

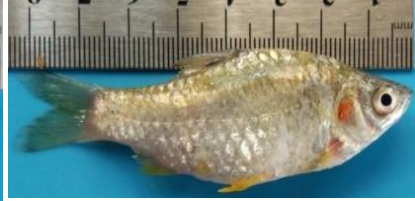

39. Puntius chola

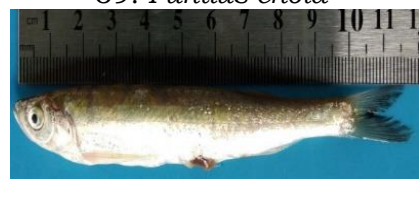

42. Salmostoma phulo

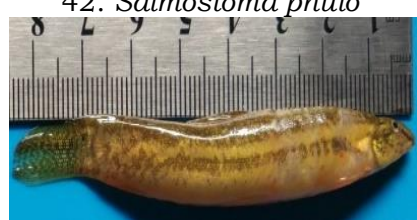

45. Lepidocephalichthys annandalei

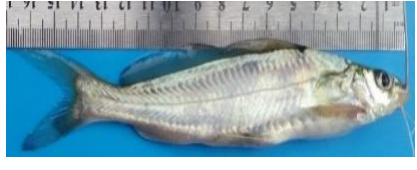

48. Mystus cavasius

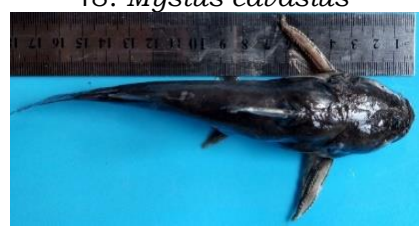

51. Rita rita

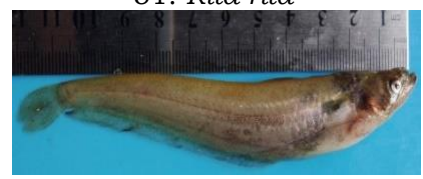

54. Ompok pabda 


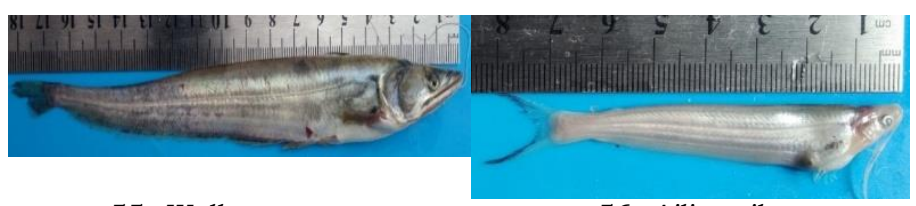

55. Wallago attu

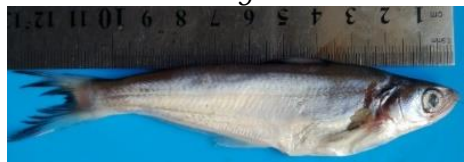

58. Eutropiichthys murius

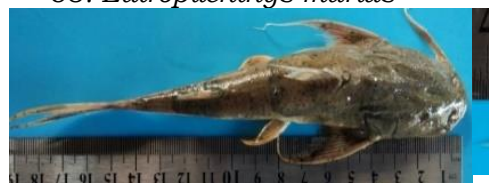

61. Bagarius bagarius

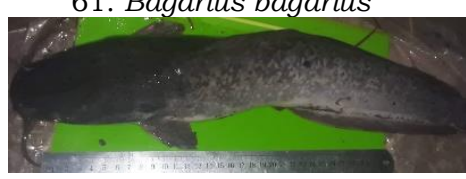

64. Clarias gariepinus

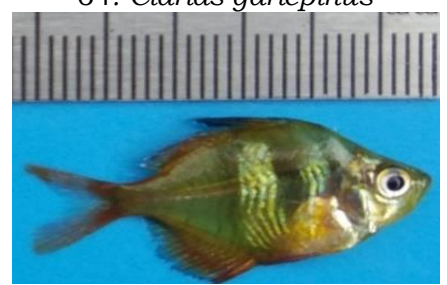

67. Parambassis lala

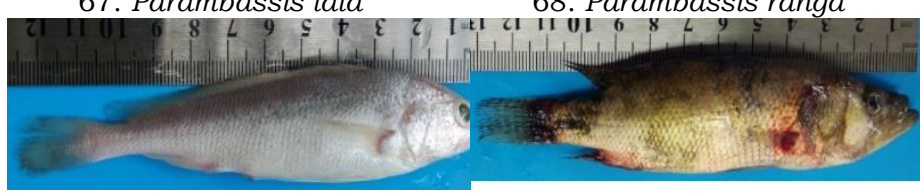

70. Otolithoides pama

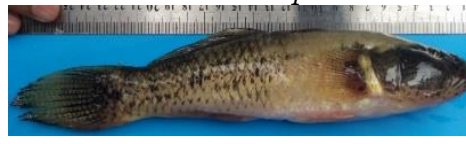

73. Eugnathogobius oligactis

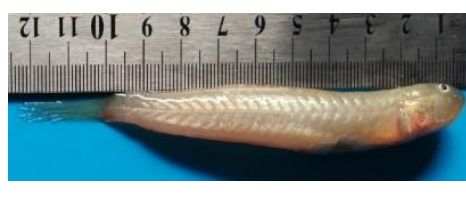

76. Pseudapocryptes elongatus

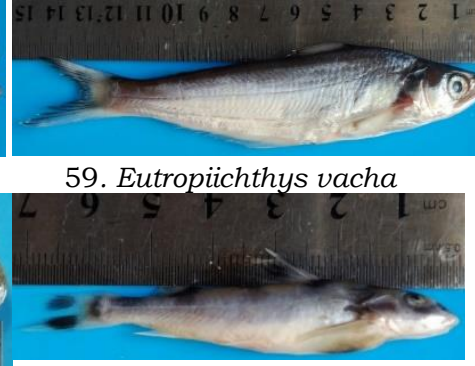

62. Gagata cenia

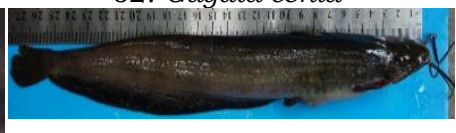

65. Heteropneustes fossilis

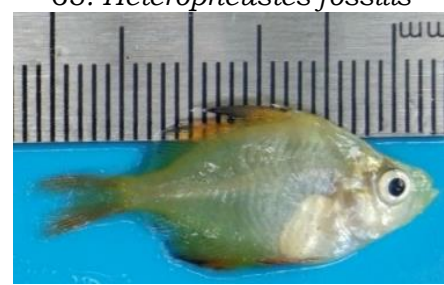

68. Parambassis ranga

71. Nandus nandus

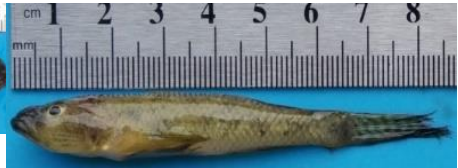

74. Glossogobius giuris

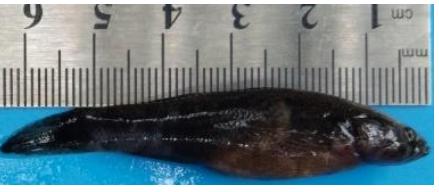

77. Eleotris lutea

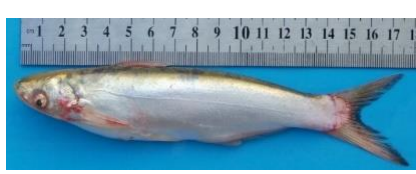

57. Clupisoma garua

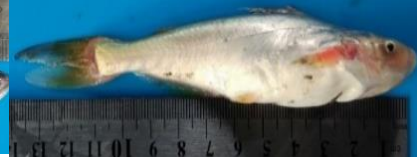

60. Pangasius pangasius

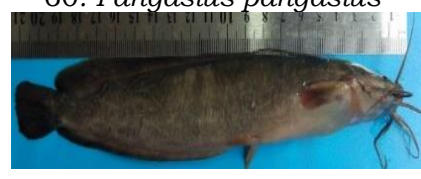

63. Clarias batrachus

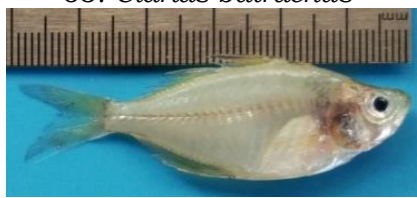

66. Chanda nama

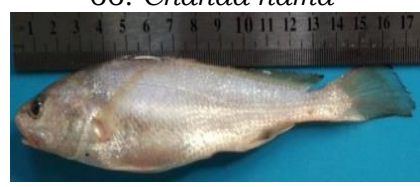

69. Johnius coitor

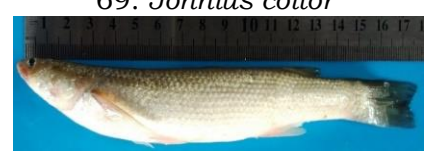

72. Rhinomugil corsula

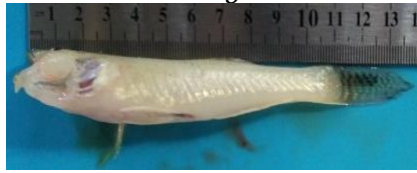

75. Oxyurichthye smicrolepis

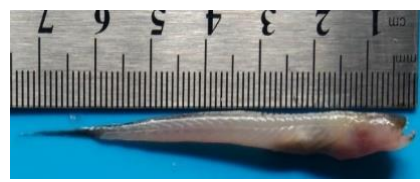

78. Odontamblyopus rubicundus 

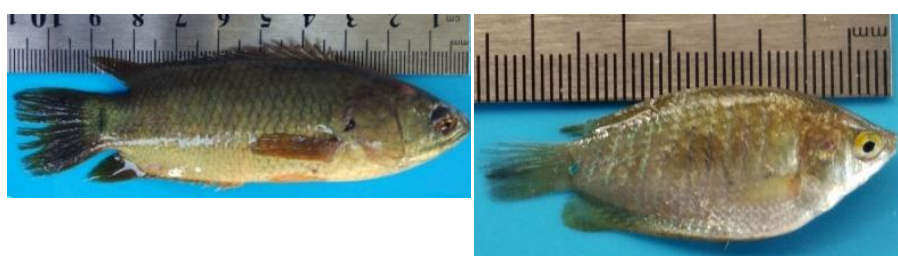

79. Anabas testudineus

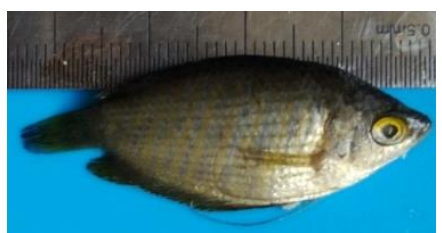

82. Trichogaster lalia
80. Trichogaster chuna

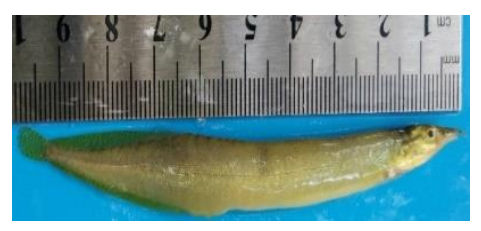

83. Macrognathus aculeatus

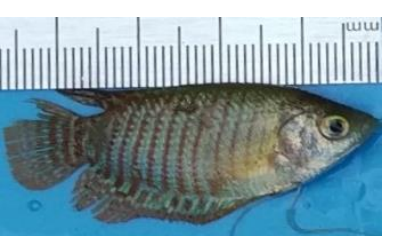

81. Trichogaster fasciatus

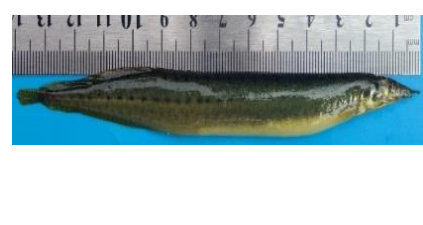

84. Macrognathus pancalus
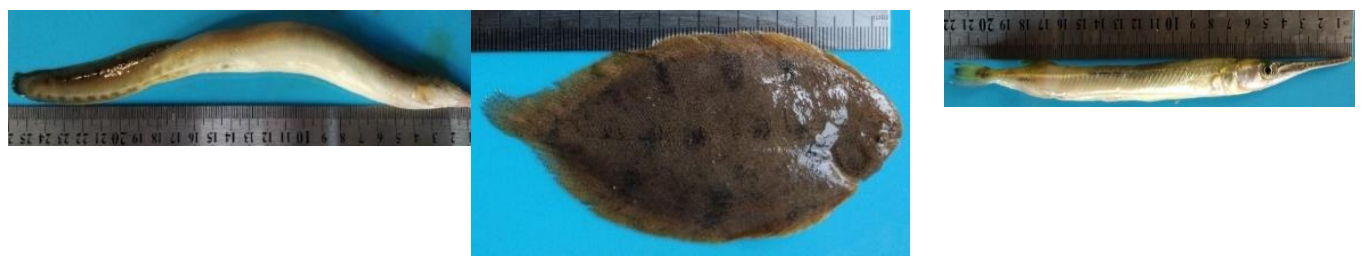

85. Mastacembelus armatus 86. Paraplagusia bilineata

87. Xenentodon cancila

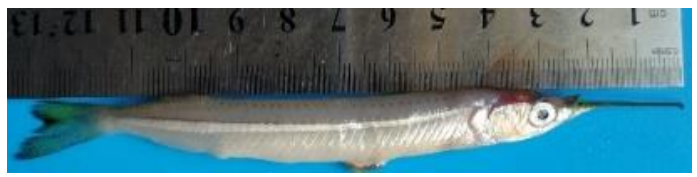

88. Hyporhamphus limbatus

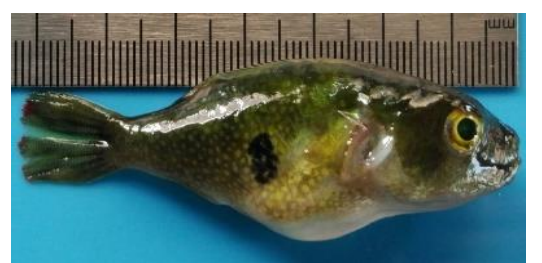

89. Leiodon cutcutia

Fig. 7-89. Photographs of the fishes recorded from Madhumati River, Magura.

distributed in number respectively in the Madhumati River. Easmin et al. (2018) found in the Jamuna River the annual values of Shannon diversity index $(H)$, Simpson index $(\lambda)$, Margalef's richness index $(D)$ and Pielou's evenness index $(E)$ as $3.350,0.053,6.931$ and 0.772 respectively. Overall values of diversity, richness and evenness indices were found as 3.717, 6.954 and 0.897, respectively by Galib et al. (2013) in Choto Jamuna River. Over all values of diversity, richness and evenness indices were found to be 1.42, 6.64 and 0.86, respectively by Rahman et al. (2015) in the Talma River. From this study and according to the experiences shared by some fishermen engaged fishing in this river, the fish biodiversity of the Madhumati River has declined with time due to over exploitation, natural causes such as flooding, siltation, drought and natural 
calamities, construction of obstacle for fish migration and breeding and lack of social awareness.

\section{CONCLUSION}

This is a preliminary and baseline study and have some limitations. A small part of the river was considered in this study which may not reflect the exact condition of the river. This study suggests the decline trend of the existing fish fauna and degrading the biodiversity. Extensive and complete study on this river along with social impact of local people may reveal the exact condition of the river.

Acknowledgement: The authors are very grateful to National Science and Technology (NST) Fellowship Program (Fiscal year 2019-2020, merit position: 492) granted by the Government of Bangladesh supporting financially to conduct the research properly.

\section{LITERATURE CITED}

AZADI, M.A. and ARSHAD-UL ALAM, M. 2013. Ichthyofauna of the River Halda, Chittagong, Bangladesh J. Zool. 41(2): 113-133.

BANGLAPEDIA, 2014. National encyclopedia of Bangladesh. Asiatic society of Bangladesh.http://en.banglapedia.org/index.php?titl=Gorai-Madhumati River.

BHUIYAN, A.L. 1964. Fishes of Dacca. Asiatic Soc. Pakistan, Publ. No. Dacca. 148 pp.

DoF, 2018. Yearbook of Fisheries Statistics of Bangladesh, 2017-18. Fisheries Resources Survey System (FRSS), Department of Fisheries. Bangladesh: Ministry of Fisheries, 2018. Volume 35

EASMIN, M.N., HABIB, A.H.M. SHAFIULLAH, AKTER, S. and MAJUMDER, K. 2018. Status and diversity of ichthyofauna of Jamuna River, Bangladesh. Jagannath University Journal of Life and Earth Sciences 4(1) 12 - 24.

FISHBASE, 2020. https://www.fishbase.se/search.php,Creative Commons AttributionNoncommercial 3.0 Unported License. (CC-BY-NC). Visited on April 2020.

GALIB, M.S., NASER, A.M.S., MOHSIN, M.B.A., CHAKI, N. and FAHAD, H.F. 2013. Fish diversity of the River Choto Jamuna, Bangladesh: Present status and conservation needs. Academic Journals 5(6): 389-395.

HOSSAIN, S.M., DAS, G.N., SARKER, S. and RAHMAN, Z.M. 2013. Fish diversity and habitat relationship with environment variables at Meghna River estuary, Bangladesh. Egyptian J. Aquatic Res. 38: 213-226.

HUSSAIN, M.G. and MAZID, M.A. 2001. Genetic improvement and conservation of carp species in Bangladesh. Bangladesh Fisheries Research Institute and International Center for Living Aquatic Resources Management, p. 74. 
IQBAL, M.M., NASREN, S., ABDULLAH-ALMAMUN, M. and HOSSAIN, M.M. 2015. Fish assemblage including threatened species in Hakaluki Haor, Sylhet, Bangladesh. J. Aqua Trop. 30(3-4): 233-246.

ISLAM, K.M., HABIB, A.K., AHSAN, E.M., ALI. M.M. and BASAK, K.S. 2016. Fish biodiversity at Sibsa River in South-Western Bangladesh: status and conservation requirements. International J. of Fisheries and Aquatic Studies 4(1): 24-28.

IUCN, 2015. Red list of threatened animals of Bangladesh Volume 5. IUCN, international Union for Conservation of Nature, Bangladesh Country Office, Dhaka, xvi, 360pp.

JOADDER, R.A.M., GALIB, M.S., HAQUE, M.M.S. and CHAKI, N. 2015. Fishes of the River Padma, Bangladesh: Current trend and conservation status. J. of Fisheries 3(2):259-266.

MARGALEF, R. 1968. Perspectives in Ecological Theory. Chicago: University of Chicago Press.

NELSON, J.S. 2006. Fishes of the World. 4th Edition, John Wiley \& Sons, Hoboken, 601 pp.

PIELOU, E.C. 1966. The Measurement of Diversity in Different Types of Biological Collections. J. of Theoretical Biology 13:131-144.

RAHMAN, A.K.A. 2005. Freshwater fishes of Bangladesh. 2nd ed., Zool. Soc. Bangladesh, Dhaka, Bangladesh. 394 pp.

RAHMAN, M.A., MONDAL, M.N., HANNAN, M.A. and HABIB, K.A. 2015. Present status of fish biodiversity in Talma River at Northern Part of Bangladesh, International Journal of Fisheries and Aquatic Studies 3(1): 341-348.

SHAFI, M. and QUDDUS, M.M.A. 1982. Bangladesher Matsho Sampad (Fisheries of Bangladesh, in Bengali). Bangla Academy, Dacca, Bangladesh. 444 pp.

SHANNON, C.E. and WEAVER, W.J. 1949. The Mathematical Theory of Communication. University of Illinois Press, Urbana, 117pp.

SIMPSON, E.H. 1949. Measurement of Diversity. Nature 163: 688.

TALWAR, P.K. and JHINGRAN, A.G. 1991. Inland Fishes of India and Adjacent Countries. OxfordIBH Publishing Co. Pvt. Ltd., New Delhi, 1158 p.

(Manuscript received on 26 November 2020 revised on 10 March 2021) 